\title{
Coherent Risk Measures and Convex Combinations of the Conditional Value at Risk (CV@R)
}

\author{
Georg Ch. Pflug \\ Department of Statistics and Decision Support Systems \\ University of Vienna
}

Dedicated to Gerhart Bruckmann on the occasion of his 70th birthday

\begin{abstract}
Summary: The conditional-value-at-risk ( $\mathbb{C V} @ R)$ has been widely used as a risk measure. It is well known, that $\mathbb{C V} @ \mathrm{R}$ is coherent in the sense of Artzner, Delbaen, Eber, Heath (1999). The class of coherent risk measures is convex. It was conjectured, that all coherent risk measures can be represented as convex combinations of $\mathbb{C V} @ \mathrm{R}$ 's. In this note we show that this conjecture is wrong.
\end{abstract}

Let the random variable $Y$ represent the future value of a portfolio. To measure the risk contained in $Y$ is an important task in stochastic finance. Among the enormous group of statistical parameters, which can be associated to $Y$, like expectation, median, variance, mean absolute deviation, coefficient of variation, Gini-measure etc., only some qualify as acceptable risk measures.

Artzner, Delbaen, Eber, Heath (1999) call a statistical parameter $\mathbb{F}(Y)$ coherent, if it has the following properties:

(i) First order stochastic monotonicity.

$P\left\{Y_{1} \leq u\right\} \geq P\left\{Y_{2} \leq u\right\}$ for all $u$ implies $\mathbb{F}\left(Y_{1}\right) \geq \mathbb{F}\left(Y_{2}\right)$

(ii) Positive homogeneity.

$$
\mathbb{F}(\lambda Y)=\lambda \mathbb{F}(Y), \quad \text { for } \lambda>0
$$

(iii) Subadditivity.

If $Y_{1}$ and $Y_{2}$ are two random variables, independent or dependent, then

$$
\mathbb{F}\left(Y_{1}+Y_{2}\right) \leq \mathbb{F}\left(Y_{1}\right)+\mathbb{F}\left(Y_{2}\right)
$$

Let $\mathcal{F}$ be the set of all coherent risk measures. Evidently, $\mathcal{F}$ is a convex set of functionals. There is practically only one known risk functional, which satisfies coherence: The conditional value-at-risk $\mathbb{C V} @$ R.

The conditional value-at-risk $\mathbb{C V} @ \mathrm{R}$ is defined as follows. Let $G$ be the probability distribution function of $Y$, i.e. $G(u)=P\{Y \leq u\}$. Then

$$
\mathbb{C V} @ \mathrm{R}_{\alpha}(Y)=\frac{1}{\alpha} \int_{0}^{\alpha} G^{-1}(u) d u
$$


It is known that $\mathbb{C V} @ \mathrm{R}_{\alpha}$ is coherent in the above sense (see Pflug, 2000). If $H$ is any monotonic, right continuous function on $[0,1]$, then

$$
\mathbb{C V} @ \mathrm{R}_{H}(Y)=\int \mathbb{C V} @ \mathrm{R}_{\alpha}(Y) d H(\alpha)
$$

is also coherent.

$\mathbb{C V} @ \mathrm{R}_{H}$ may be represented as

$$
\begin{aligned}
\mathbb{C V} @ \mathrm{R}_{H}(Y) & =\int_{0}^{1} \int_{0}^{1} \frac{1}{\alpha} \mathbb{1}_{[0, \alpha]}(u) G^{-1}(u) d H(\alpha) d u \\
& =\int_{0}^{1} \int_{u}^{1} \frac{1}{\alpha} d H(\alpha) G^{-1}(u) d u \\
& =\int_{0}^{1} h(u) G^{-1}(u) d u
\end{aligned}
$$

Here

$$
h(u)=\int_{u}^{1} \frac{1}{\alpha} d H(\alpha) .
$$

$h$ is monotonically decreasing in $u$ and conversely, every monotonically decreasing function $h$ has a representation (2). Thus the convex hull of all $\mathbb{C V} @ \mathrm{R}_{\alpha}$ is given by all risk measures of the form (1).

It was proved by Uryasev and Rockafellar (2000) that

$$
\mathbb{C V} @ \mathrm{R}_{\alpha}(G)=\max \left\{a-\frac{1}{\alpha} \int[u-a]^{-} d G(u): a \in \mathbb{R}\right\}
$$

It was conjectured that the class $\mathbb{C V} @ \mathrm{R}_{H}$ coincides with the class of coherent risk measures. This is however not true: Consider the new measure

$\mathbb{F}^{*}(G)=\max \left\{\left(a_{1}+a_{2}\right) / 2-\frac{1}{2 \alpha_{1}} \int\left[u-a_{1}\right]^{-} d G(u)-\frac{1}{2 \alpha_{2}} \int\left[u-a_{2}\right]^{-} d G(u): a_{1} \geq \lambda a_{2}\right\}$

Proposition. The risk measure (3) is coherent, but is not of the form (1).

Proof. Let $H\left(a_{1}, a_{2}, Y\right)=\left(a_{1}+a_{2}\right) / 2-\frac{1}{2 \alpha_{1}} \int\left[u-a_{1}\right]^{-} d G_{Y}(u)-\frac{1}{2 \alpha_{2}} \int\left[u-a_{2}\right]^{-} d G_{Y}(u)=$ $\left(a_{1}+a_{2}\right) / 2-\frac{1}{2 \alpha_{1}} \mathbb{E}\left(\left[Y-a_{1}\right]^{-}\right)-\frac{1}{2 \alpha_{2}} \mathbb{E}\left(\left[Y-a_{2}\right]^{-}\right)$.

$\mathbb{F}^{*}\left(G_{Y}\right)=\inf \left\{H\left(a_{1}, a_{2}, Y\right): a_{1} \geq \lambda a_{2}\right\}$. Since $H\left(a_{1}, a_{2}, Y\right)$ is stochastically monotone in $Y$, the same is true for $\mathbb{F}^{*}$, i.e. (1) is fulfilled. Since for all $\mu>0$, $H\left(\mu a_{1}, \mu a_{2}, \mu Y\right)=\mu H\left(a_{1}, a_{2}, Y\right)$ and since also the condition $a_{1} \geq \lambda a_{2}$ is homogeneos, $\mathbb{F}^{*}$ is positively homogeneous. Finally $H\left(a_{1}, a_{2}, X+Y\right) \leq H\left(a_{1} / 2, a_{2} / 2, X\right)+$ $H\left(a_{1} / 2, a_{2} / 2, Y\right)$. and this implies that $\mathbb{F}^{*}$ is subadditive.

If the constraint $a_{1} \geq \lambda a_{2}$ was not present, the infimum would be

$$
\frac{1}{2} \mathbb{C V} @ \mathrm{R}_{\alpha_{1}}(Y)+\frac{1}{2} \mathbb{C V} @ \mathrm{R}_{\alpha_{2}}(Y)
$$

However, the constraint does not change the property of coherence, but introduces a new type of measure. 
Let us finally remark, that if one weakens the assumption of monotonicity w.r.t. first order stochastic dominance by the assumption of second order stochastic dominance (see Fishburn, 1980)

(i') $\int_{-\infty}^{v} P\left\{Y_{1} \leq u\right\} d v \geq \int_{-\infty}^{v} P\left\{Y_{2} \leq u\right\} d v$ for all $v$ implies $\mathbb{F}\left(Y_{1}\right) \geq \mathbb{F}\left(Y_{2}\right)$, then more functionals satisfy (i'),(ii),(iii). Examples are

$$
\mathbb{F}(Y)=\mathbb{E}(Y)-\sqrt{\mathbb{E}\left([Y-\mathbb{E} Y]^{-}\right)^{2}}
$$

or

$$
\mathbb{F}(Y)=\mathbb{E}(Y)-\frac{1}{2} \mathbb{E}(|Y-\mathbb{E} Y|)
$$

\section{Literatur}

P. Artzner, F. Delbaen, J.-M. Eber, and D. Heath. Coherent measures of risk. Mathematical Finance, 9:203-228, 1999.

P.C. Fishburn. Stochastic dominance and moments of distributions. Mathematics of Operations Research, 5:94-100, 1980.

G. Pflug. Some remarks on the Value-at-Risk and the conditional Value-at-Risk. In S. Uryasev, editor, Probabilistic Constrained Optimization - Methodology and Applications, pages 272-281, Kluwer Academic Publishers, 2000.

S. Uryasev and R.T. Rockafellar. Optimization of conditional Value-at-Risk. The Journal of Risk, 2(3):21-41, 2000.

S. Uryasev. Conditional Value-at-Risk: Optimization algorithms and applications. $\mathrm{Fi}^{-}$ nancial Engineering News 14, February 2000.

Author's address:

Prof. Dr. Georg Ch. Pflug

Department of Statistics and Decision Support Systems

University of Vienna

Universitätsstraße 5

A-1010 Wien, Austria

E-mail: georg.pflug@univie.ac.at 